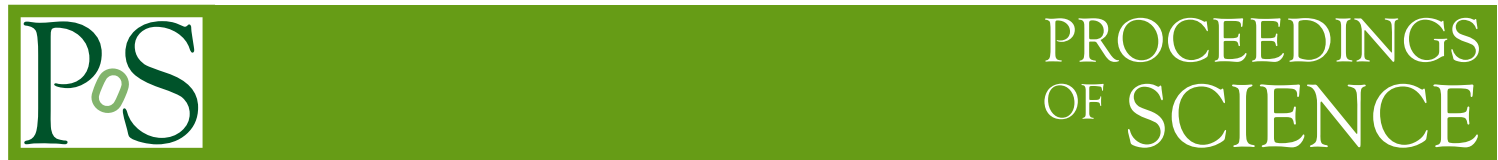

\title{
Nuclear structure and stellar weak interaction rates of nuclei below the ${ }^{132} \mathrm{Sn}$ core
}

\section{Saha Sarkar*}

Nuclear and Atomic Physics Division, Saha Institute of Nuclear Physics, Kolkata 700064, INDIA

E-mail: maitrayee.sahasarkar@saha.ac.in

\section{S. Sarkar}

Department of Physics, Bengal Engineering and Science University, Shibpur, Howrah 711103, INDIA

E-mail: ss@physics.becs.ac.in

Shell model calculations have been done for a few valence particle and hole isotopes of $S n$ above ${ }^{100} \mathrm{Sn}$ and below ${ }^{132} \mathrm{Sn}$ cores using a CD-Bonn potential based interaction. A few matrix elements of this realistic interaction have been modified by using experimental data of the two valence neutron $S n$ isotope above ${ }^{100} \mathrm{Sn}$. Calculations with this modified interaction reproduces the enhanced $\mathrm{B}\left(E 2,0^{+} \rightarrow 2^{+}\right)$values of lighter $S n$ isotopes. But it seems that the interaction strength suitable for nuclei close to $N=Z{ }^{100} S n$ may differ from that appropriate for isotopes near neutron-rich ${ }^{132} \mathrm{Sn}$.

10th Symposium on Nuclei in the Cosmos

July 27 - August 12008

Mackinac Island, Michigan, USA

\footnotetext{
* Speaker.
} 


\section{Introduction}

With the advent of newer and more sophisticated experimental devices and techniques, the empirical knowledge about nuclei in the entire nuclear chart is being expanded at a fast rate. New data are coming up, throwing new challenges to the existing theories. But experimental travel to these untrodden territories are extremely difficult and involve large errors in the measurements. Most of the exotic nuclei are still not accessible for spectroscopic studies. So the experimental ventures also have to rely intensely on theoretical predictions. Experimental information about $S n$ isotopes span a large domain - starting from ${ }^{100} S n$, a proton rich nucleus, to ${ }^{138} S n$, which has large neutron excess. Ten stable isotopes of $S n$ are found in nature. So the isotopes of $S n$ offer theoreticians a novel opportunity to test their theories and predict new problems for experiments.

In the present work, shell model calculations have been done using a CD-Bonn potential based interaction to study a few valence neutron particle and hole isotopes of $S n$. The calculations have been done using both particle and hole spaces by taking the ${ }^{100} S n$ and ${ }^{132} S n$ as respective cores.

Very recently, substantial efforts are being spent to determine the $\mathrm{B}\left(E 2,0^{+} \rightarrow 2^{+}\right)$values of the $S n$ isotopes over the whole range of $S n$-masses. For a sequence of nuclei between two shell closures, it is expected that these B(E2) values should follow a parabolic trend which peaks at midshell. But it has been found that the trend in even-even $S n$ isotopes is not symmetric with respect to the mid-shell mass number $A=116$. There have been several efforts to explain this anomaly. In Ref. [1], this asymmetry was somewhat explained within large scale shell model with contributions from $1 g_{9 / 2}$ protons from the $\mathrm{Z}=50$ core. But even the enlarged calculations deviated from the new experimental data [2] for lighter $S n$ isotopes. This observation showed that further core-polarization effects may be needed and/or a better effective interaction should be introduced [3]. Alpha-like correlations were also thought to be important for explaining this feature [4]. Recently, it has been conjectured that the "so-called monopole drift" of the single-particle orbits can play a key role in self conjugate nuclei [2]. But the observed increase in transition strength for light isotopes of $S n$ near ${ }^{100} S n$ clearly shows the need for further theoretical investigations of the nucleon-nucleon interaction in this region [2].

In the present work, the realistic interaction has been marginally modified and the B(E2) values in a few valence particle and hole isotopes of $S n$ above ${ }^{100} S n$ and below ${ }^{132} S n$ cores have been studied to understand this problem from a different perspective.

\section{Shell model calculations}

The shell model (SM) calculations were carried out in the sn100pn model space with the sn100pn Hamiltonian [5] using the windows version of OXBASH [6] and Nushell@ MSU [7] codes. There are three parts in this Hamiltonian which are the proton-proton (sn100pp), neutronneutron (sn100nn), and proton-neutron (sn100pn) interactions along with the Coulomb interaction $(s n 100 \mathrm{co})$ between the protons. But as we are interested in $S n$ isotopes only, the neutron-neutron part, i.e. $s n 100 n n$ was only relevant for our calculations.

The calculations have been done using both particle and hole spaces by taking the ${ }^{100} \mathrm{Sn}$ and ${ }^{132} \mathrm{Sn}$ as respective cores. The few valence particle/hole nuclei near these doubly closed magic cores are described in the valence space consisting of $\left(1 g_{7 / 2}, 2 d_{5 / 2}, 2 d_{3 / 2}, 3 s_{1 / 2}\right.$ and $\left.1 h_{11 / 2}\right)$ orbitals for 
proton particles and neutron $\left(1 g_{7 / 2}, 2 d_{5 / 2}, 2 d_{3 / 2}, 3 s_{1 / 2}\right.$ and $\left.1 h_{11 / 2}\right)$ orbitals for neutron particles or holes.

Until recently, the energies of the single particle orbitals (spes) of the valence space above the ${ }^{100} \mathrm{Sn}$ core were not measured experimentally. So the choice of spes [8] can be a substantial source of uncertainty in these calculation. For the single hole energies [5] the energy levels observed in ${ }^{131} \mathrm{Sn}$ have been used.

In a recent work Ref. [9], the energy difference between single-neutron $1 g_{7 / 2}$ and $2 d_{5 / 2}$ orbitals has been estimated. So the single particle energies above ${ }^{100} \mathrm{Sn}$ has been determined keeping in mind the following observations. Firstly, energy difference between $1 g_{7 / 2}$ and $2 d_{5 / 2}$ is $171 \mathrm{keV}$. Secondly, binding energy of ${ }^{101} \mathrm{Sn}_{\text {g.s. }}$. with respect to ${ }^{100} \mathrm{Sn}$ is $10.851 \mathrm{MeV}$ [10].

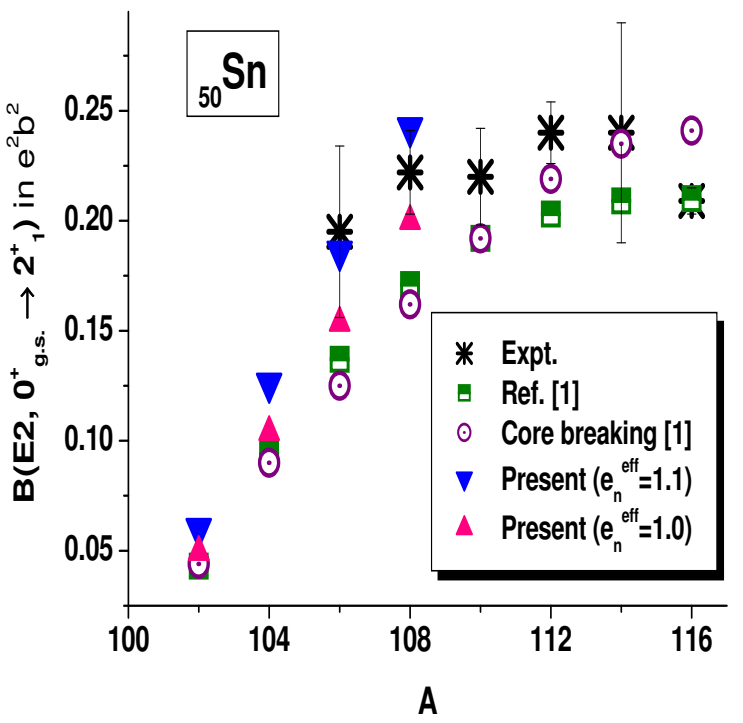

Figure 1: Comparison of calculated and experimental $\mathrm{B}\left(E 2,0^{+} \rightarrow 2^{+}\right)$values below the midshell.

Previous two body matrix elements for $n-n$ interaction were multiplied by a factor of 0.9 in Ref. [5] to improve results for ${ }^{130} \mathrm{Sn}$. In the present work, we have multiplied the two body matrix elements for $n-n$ interaction ( $2^{+}$terms) by 1.275 to reproduce the $2_{1}^{+}$energy of ${ }^{102} S n$.

\section{Results and Discussions}

With the modified interaction, we have calculated the binding energies, $\mathrm{E}\left(2_{1}^{+}\right)$and the $\mathrm{B}\left(E 2,0^{+} \rightarrow 2^{+}\right)$values of ${ }^{102-108} S n$ and ${ }^{126-130} \mathrm{Sn}$ (Table 1). The results with the CD-Bonn potential based interaction are also shown for comparison. For ${ }^{126-130} \mathrm{Sn}$, calculations have been done taking both (i) ${ }^{100} \mathrm{Sn}$ and (ii) ${ }^{132} S n$ as the cores. For ${ }^{102-108} S n$, the results show good agreement with data with both the interactions. The modified interaction gives some improvement in agreement. For ${ }^{126-130} S n$, with ${ }^{100} S n$ as the core, binding energy with respect to the core is over- predicted with the modified interaction. But for ${ }^{132} \mathrm{Sn}$ core, with single particle energies are taken from the experimental spectra of ${ }^{131} \mathrm{Sn}$, the binding energy shows substantial improvement. The excitation energies of the $2_{1}^{+}$states

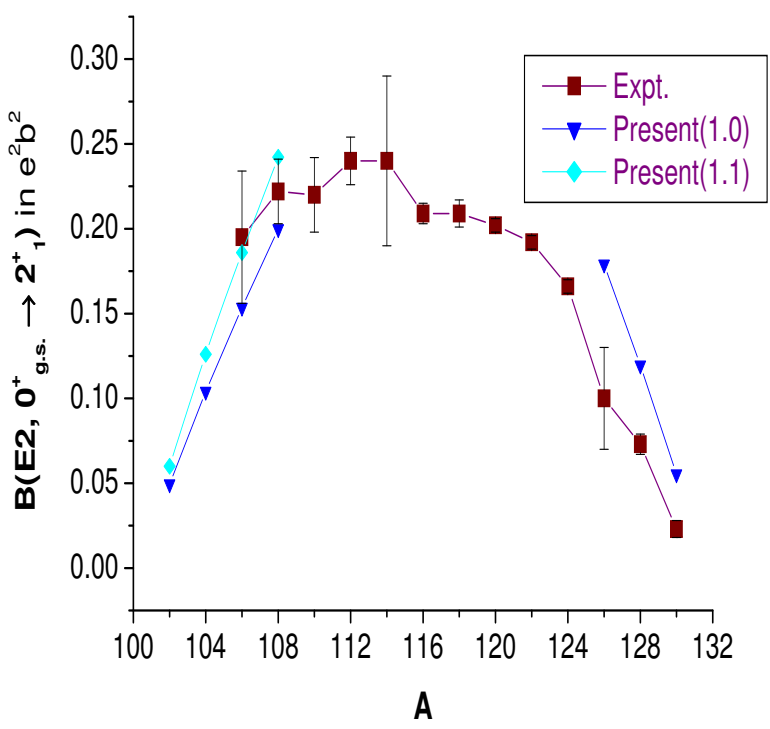

Figure 2: Comparison of calculated and experimental $\mathrm{B}\left(E 2,0^{+} \rightarrow 2^{+}\right)$values between ${ }^{100} S n$ and ${ }^{132} \mathrm{Sn}$. 


\begin{tabular}{ccccccc}
\hline Isotope & \multicolumn{3}{c}{ Binding Energy w.r.t. ${ }^{100}$ Sn } & \multicolumn{3}{c}{ Excitation energy of $2_{1}^{+}$} \\
& Expt. & CD-Bonn & Present & Expt. & CD-Bonn & Present \\
\hline${ }^{102} S n$ & 24.293 & 23.385 & 24.323 & 1.472 & 1.683 & $1.468^{1}$ \\
${ }^{104} S n$ & 47.095 & 46.052 & 48.005 & 1.260 & 1.495 & 1.258 \\
${ }^{106} S n$ & 69.078 & 67.980 & 70.939 & 1.208 & 1.414 & 1.156 \\
${ }^{108} S n$ & 89.931 & - & 93.075 & 1.206 & - & 1.111 \\
${ }^{130} S n$ & 265.498 & 270.527 & 284.174 & 1.221 & 1.559 & 1.194 \\
& $(-11.187)^{2}$ & $(-13.44)^{2}$ & $(-12.260)^{2}$ & & & \\
${ }^{128} S n$ & 252.552 & 257.974 & 270.472 & 1.169 & 1.357 & 1.069 \\
& $(-25.504)^{2}$ & $(-26.127)^{2}$ & $(-25.108)^{2}$ & & & \\
${ }^{126} S n$ & 239.094 & 270.527 & 284.174 & 1.141 & 1.270 & 0.959 \\
& $(-38.962)^{2}$ & $(-39.583)^{2}$ & $(-38.637)^{2}$ & & & \\
\hline
\end{tabular}

${ }^{1}$ Normalised; ${ }^{2}$ With respect to ${ }^{132} \mathrm{Sn}$

Table 1: Comparison of theoretical results with experimental data. Energies are in MeV.

for ${ }^{102-108} \mathrm{Sn}$ is reproduced better by the modified interaction, but for modified interaction, the $\mathrm{E}\left(2_{1}^{+}\right)$values of ${ }^{126-130} \mathrm{Sn}$ show much faster decrease with increase in the number of holes.

$\mathrm{B}(\mathrm{E} 2)$ values have been obtained with effective neutron charges $\left(e_{n}^{\text {eff }}\right)$ as 1.0 and 0.5 in the results from [1] and core-breaking calculations[1], respectively. We have used $\left(e_{n}^{e f f}\right)$ as 1.0 and 1.1 in our work. The results for lighter isotopes definitely show improvements (Fig. 1) compared to previous results. But if we look at the total range (Fig.2), we find that this interaction has overpredicted $\mathrm{B}(\mathrm{E} 2)$ values of heavier $S n$ isotopes.

To reproduce the data for the $S n$ isotopes close to $N=Z{ }^{100} \mathrm{Sn}$, an increase in interaction strength is required which results in enhanced configuration mixing and larger $\mathrm{B}(\mathrm{E} 2)$ values. But in the region just below ${ }^{132} \mathrm{Sn}$, isotopes are neutron rich. This interaction adjusted to reproduce the data near ${ }^{100} \mathrm{Sn}$ predicts enhanced $\mathrm{B}(\mathrm{E} 2)$ for heavier isotopes also. So the interaction is unable to predict the asymmetric nature of the $\mathrm{B}(\mathrm{E} 2)$ trend.

The properties of ${ }^{130} \mathrm{Sn}$ and its neighbouring nuclei are important for r-process nucleosynthesis. The astrophysical beta decay rates are important in this respect. So we started calculation of the $\mathrm{B}(\mathrm{GT})$ strength distribution for

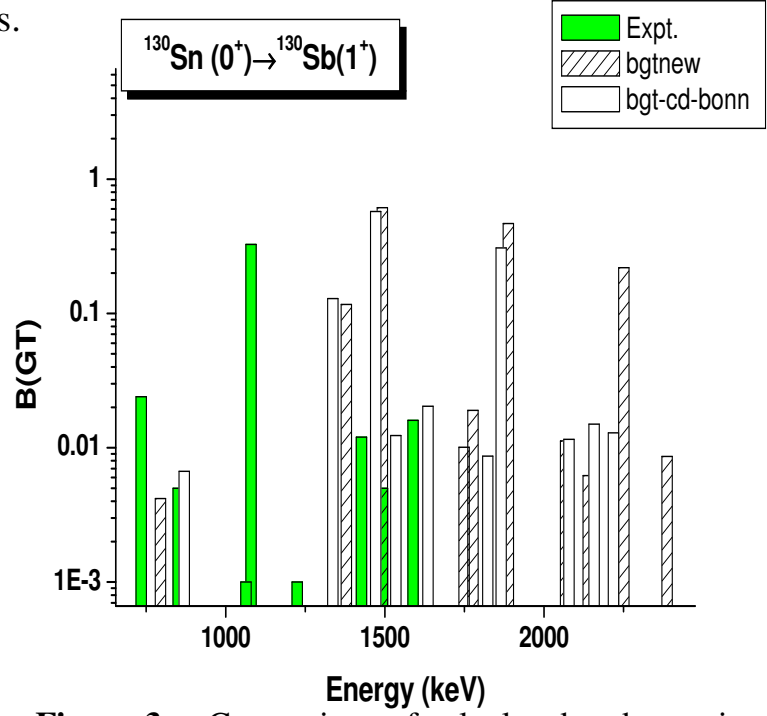

Figure 3: Comparison of calculated and experimental $\mathrm{B}(\mathrm{GT})$ values for beta decay from ${ }^{130} \mathrm{Sn}$ to ${ }^{130} \mathrm{Sb}$ ${ }^{130} \mathrm{Sn}$ using the original CD-Bonn potential based interaction from Brown et al. [5] and the modified version. Theoretical results are shown without any quenching (Fig.3). Experimental data are from Ref. [11]. Theoretical results show large shift in energy centroid of the distribution compared to the exptl. data. Such large shift in strength distribution may lead to unphysical beta decay rates. 
It shows that the interactions definitely need improvement for better predictability and application in astrophysical scenario.

\section{Conclusion}

The interactions used in the study of $S n$ isotopes predict some of the properties like $\mathrm{E}\left(2_{1}^{+}\right)$ energies reasonably well. But they fail to explain the transition rates. A slightly modified CDBonn potential based interaction shows some improvement in the predictions of the $\mathrm{B}(\mathrm{E} 2)$ values for light $\mathrm{Sn}$ isotopes. Binding energies and $\mathrm{E}\left(2_{1}^{+}\right)$energies are reasonable for light isotopes. But the modified interaction fails for isotopes close to ${ }^{132} \mathrm{Sn}$. The theoretical calculations for $\mathrm{B}(\mathrm{GT})$ distribution using both the interactions can not reproduce the experimental trend. But the theoretical studies face several problems, e.g., lack of experimental data for unambiguous determination of single particles energies. This is valid for both neutron particle (near ${ }^{100} \mathrm{Sn}$ the problem is more serious) and hole energies. The whole range of $S n$ isotopes needs more comprehensive study. It seems that the interaction strength suitable for nuclei close to $N=Z{ }^{100} S n$ may differ from that appropriate for isotopes near neutron-rich ${ }^{132} \mathrm{Sn}$. For reliable predictability in astrophysical scenarios the interaction developed from realistic $\mathrm{N}-\mathrm{N}$ interactions should be fine-tuned to local systematic.

\section{References}

[1] A. Banu et al., ${ }^{108}$ Sn studied with intermediate-energy Coulomb excitation, Phys. Rev. C 72, 061305 (R) (2005).

[2] A. Ekström et al., $O_{g s}^{+} \rightarrow 22_{1}^{+}$Transition strengths in ${ }^{106}$ Sn and ${ }^{108}$ Sn, Phys. Rev. Lett. 101, 012502 (2008).

[3] J. Cederkäll et al., Sub-Barrier Coulomb excitation of ${ }^{110}$ Sn and its implications for the ${ }^{100}$ Sn shell closure, Phys. Rev. Lett. 98, 172501 (2007).

[4] C. Vaman et al., $Z=50$ shell gap near ${ }^{100}$ Sn from intermediate - energy Coulomb excitations in even mass ${ }^{106-112}$ Sn isotopes, Phys. Rev. Lett. 98, 162501 (2007).

[5] B.A. Brown, N. J. Stone, J. R. Stone, I. S. Towner, and M. Hjorth-Jensen, Magnetic moments of the $2_{1}^{+}$states around ${ }^{132}$ Sn, Phys. Rev. C 71, 044317 (2005).

[6] B.A. Brown et al., Oxbash for Windows PC,MSU-NSCL ReportNo. 1289, (2004).

[7] B. A. Brown and W. D. M. Rae, Nushell@MSU, MSU-NSCL report (2007).

[8] Morten Hjorth-Jensen, Thomas T.S. Kuo, Eivind Osnes, Realistic effective interactions for nuclear systems, Phys. Rep. 261, 125 (1995).

[9] D. Seweryniak et al., Single - Neutron states in ${ }^{101}$ Sn, Phys. Rev. Lett. 99, 022504 (2007).

[10] G. Audi, A. H. Wapstra, C. Thibault, The 2003 atomic mass evaluation: (II). Tables, graphs and references, Nucl. Phys. A 729, 337 (2003).

[11] http://www.nndc.bnl.gov 